\title{
The strategy of teaching the culture of debating in FL to non-linguist students
}

\author{
Elena Komochkina ${ }^{1,{ }^{*}}$, Tatiana Selezneva ${ }^{1}$, and Natalia Akimova ${ }^{1}$ \\ ${ }^{1}$ Moscow Region State University, Moscow region, Mytishchi, 24, Str. Vera Voloshina, Russia, \\ 141014
}

\begin{abstract}
The article considers some contextual, interdisciplinary and linguistic aspects of developing non-linguistic students' speaking skills necessary for cross-cultural academic communication, with special attention being paid to the their abilities to master the culture of debating, acquiring specific features of their future profession through learning specialized vocabulary and using a foreign language as an effective tool to reach the professional goals. The proposed strategy of FL communication teaching is rooted in the basics of D. Dorner's situation integrity' theory, on the one hand, taking into account discovery patterns of science undergraduates' educational activity, on the other, and cognitive aspects of academic discourse as well with an emphasis on acquiring professional interlanguage dialogic skills. The proposed course of study is meant to master the professional speaking skills of science undergraduates. The algorithm of teaching has been tested in FL classes with Maths and Physics students of MGOU (Moscow Region State University) during 2019/2020 academic year and obtained the positive approval of FL teaching staff.
\end{abstract}

\section{Introduction}

The rapid growth of globalization along with the apparent recent trend to integration of the key axiological aspects of modern life, i.e. language, cognition, life philosophy and universal cultural human values, exhibit the closer interaction between people of different nationalities, cultures and professional communities as well as diversity in means of expression (personal, interpersonal, intragroup, intergroup, mass communication). These types of communication could take place in different spheres of life from cultural, scientific to recreational and professional. The ongoing changes in communicative patterns and their range extension could not but help influencing educational policy and furthering processes of universalization and standardization in professionally oriented instruction $[1,2,3,4,5,6]$.

It is a matter of common knowledge that foreign language teaching for special purposes is directly related to political, economic, social and cultural changes. Researchers in education accentuate the centrality of a person and humanitarian sphere since progress in science and education enables people to impact deliberately on the society development. Interdisciplinary studies register the alterations in the value system and its introduction or adaptation into one or another world view $[1,7,8]$.

* Corresponding author: komochkina2010@yandex.ru 
In recent years scientists have been more than explicit about evolutionary processes in educational environment such as integration, personalization and differentiation in some profiles of training whose subject matter tend to be more fundamental and of interdisciplinary character while the processes of teaching are becoming more algorithmically organized and student-oriented $[3,7,9]$.

According to the social construction theory introduced by sociologists P. Berger and Th. Luckmann the world view is determined by different social contexts in which the notions of "reality" and "knowledge" should not be taken for granted and their interrelationships are to be examined by specialists in sociolinguistics and cultural studies [10].

Since any "reality" and any "knowledge" which affect the world view and cultural values are subjected to constant change and could be transferred and preserved in socially and professionally relevant contexts, the sociology of knowledge investigates these processes, the methodology of science attempts to explain the regular patterns of interaction between cognition and language while the FLT (Foreign Language Teaching) aims to put these theories into practice $[6,11,12,13]$.

These many-faceted processes have paved the way to linguistic education taking various forms of verbal communication such as polemics, disputes, debates, discussions, round tables, symposiums and conferences.

Theoretical and practical basics of communication in L2 teaching traditionally accounted for the following factors, i.e., proficiency in vocabulary, grammar and composition skills in both native and foreign languages $[7,9,14]$. The present research is aimed to examine the characteristic features of the formation of effective communication skills in accordance with the principles of knowledge structuring in professionally determined contexts and academic research activity of students as well as cognitive nature of linguistic matter. The study is also targeted to develop a training course to accompany the teaching aids.

The numerous studies consider a FL (Foreign Language), on the one hand, as an integral element of ethnocultural heritage having accumulated the precedent communication experience, on the other, as a tool for exchanging professionally relevant information.

It is noteworthy that current research in methodology observes some excessive "technicality", standardization and unification of present-day special FL education in tertiary training of non-linguist students, which could not be considered beyond a shadow of doubt. The German philosopher M.Heidegger warned us against the trend of reduction in cognitive activity by writing that the threat to humanity comes not as much from wars or degradation or nature destruction but from the fact that people discontinued thinking and that instead of thinking science measures, calculates and applies thus turning Being as such into entity [15].

Nevertheless, with bearing in mind Heidegger's threat, the "technicality" of educational process might have manifold implications; on the one hand, it organizes the procedures underlying teaching and learning routes, on the other, it is supposed to make students' creative and reproductive activity more effective.

In accordance with methodological theory the acquisition of technology (some algorithm, or an instrumental tool) of professional communication by involvement into dynamic educational cognitive activity is an indispensable requirement to be pursued for a future specialist training [4,11].That is why, the most sought after practices in FLT are considered to be - making presentations, designing a case study, participating in didactic games and problem-solving lectures in which a student is not only a passive listener but he or she is also involved in logical argumentation and finding an answer to a question posed by a lecturer. In other words, the algorithmization of teaching communication in FL might foster the mastering of speaking skills due to the very nature of the discipline FLT $[4,6]$. 
The participation of students in "round tables", debates and discussions teaches them to uncover their creative selves, make autonomous judgements, get acquainted with the culture of discussion and develop critical thinking. Along with mastering speaking and discussion skills as well as abilities to deliver a full-scale speech to the audience, this educational activity contributes to the development of communicative competence. As a whole these skills and competences would enable the future specialist to engage in professional communication.

FL educationalist, teaching methodology experts, language teachers, however, are much preoccupied nowadays with the problem that the present system of language education is marked by the controversy over the current goals of training a highly-skilled professional in science and inefficiency of the existing special language teaching programmes which generally are not aimed at participation in cross-cultural academic communication. The study of the relevant sources in pedagogy, psychology and foreign language teaching revealed the objective and subjective reasons for the controversy. Above all, the common age-old vocabulary and grammar issues along with compositional and structural deficiencies as well as underdeveloped culture of debating are believed to aggravate the situation.

In view of these issues we propose a strategy of FL communication teaching which is rooted in the basics of D. Dorner's situation integrity' theory, on the one hand, taking into account discovery patterns of science undergraduates' educational activity, on the other, and cognitive aspects of academic discourse as well with an emphasis on acquiring professional interlanguage dialogic skills.

So the final aim of the present research was to elaborate an algorithm (strategy) of teaching oral speech professional utterances to non-linguistic students based on the theoretically proved concepts and try it in an Fl course of studies during one term in 20192020.

\section{Methodology. The culture of debating as a key to professional competency in an academic community}

The culture of professional discussing is determined by several factors which presuppose the integrity of perception of professional communication context. These factors could be broken into two groups, i.e., internal (personal) and external. The latter include communication contexts, political and economic relations between countriescommunicants, nationally conditioned boundaries of cross-cultural communication, the character of the target audience, topics and the personality of communicants.

The internal factors might entail the background knowledge about the mental and cognitive sphere underlying the educational system in the country-communicant, the level of competency in the subject under discussion, in particular, and in the professionally oriented situation of communication as a whole, presentational skills and abilities, some objective difficulties which students might face as well as readiness and willingness to participate in communication.

Whether these factors be accounted for or not in FLT of non-linguist students depends on the efficiency of preparatory work, arranging and managing of such internationally held events as conferences, seminars, workshops, negotiations.

\subsection{Integrity of a professional situational context}

As a form of activity targeted at passing information, knowledge and conveying meaning, communication pursues some definite goals. That is why, misinterpretation of a 
professional situation, the wrong choice of presentational forms and content, underestimation of possible controversies between communicants and parties involved might lead to the failure of communication.

According to D. Dorner, the wide variety of situations evolving in communication (simple, complex, conventional, unpredictable, unexpected etc.) might need a variety of methodical techniques and approaches to deal with different ways of presenting vocabulary and grammar items, semantization and, the last but not the least, mastering the strategies of structuring the subject matter of an utterance [16].

The aim of communication being transparent, the wide-known ways of structuring knowledge concerning the situation in question might be used, i.e., the definition of the purpose of communication, the choice of discursive strategies and verbal means with an emphasis on special terminology and general scientific terms, its probable development, the expected outcome of the given situation accounting for addressee's involvement $[9,14]$. Moreover, the approach to structuring the knowledge about the situation might imply its present-day meanings, expedience, appropriateness, its practical and theoretical significance. In compliance with Dorner's ideas a situation of communication might be considered in terms of its dynamic development. As a result, information, i.e., context and verbal material, is better perceived when it is deployed without interruption taking into account its precedent, if its possible scenario might be anticipated by means of extrapolation the available data into the future contexts [16]. The expedient situation of communication in FLT might be presented as a problem-solving situation with all abovementioned properties to be considered. The deployment of the scenario under consideration at FL lessons implies the solution of a certain problem which is understood as one of the most effective approaches in contemporary methodology.

"As far as the training of a new generation of specialists is concerned linguodidactic tools of teaching ought to be directly correlated to the modelling of contexts and combined with conventional educational resources"[6]. The English language text-book "Insight into the structure" by O. Klochkova and E.Komochkina and "Academic Vocabulary in Use. Vocabulary reference and practice» by McCarthy M., O'Dell F. might provide an example of knowledge structuring as well as context designing within the programme of FLT to master's applicants specializing in Theoretical Physics [17,18]. The subject matter conforms to the following structuring, e.g., reporting on some research in one's field of investigation, describing types of research methods, limiting and specifying an argument, expressing points of view, elaborating aims, developing criteria for the definition and evaluation of investigation, presenting a new explication of creative methods, providing an infrastructure for undergraduate study, contributing to the development of the discipline under study, etc. [18].

In addition to knowledge structuring the programme also provides a vast variety of scenarios (namely, professional situations) aimed at identification, evaluation, synthesis, modelling and students' awareness raising of the current global contexts of professional communication such as fundamental physical forces, the standard model, the measurement theory, state-of-the art models of the universe, fundamental structure of matter, quantum computing etc.

It is the initiation of students into the professional world that constitutes the basics of the programme.

The experience of teaching provides evidence that students need "to be prepared for integration into professional environment, professional contexts, which implies projection of subject and social contexts into present-day educational content. Students learn professional discourse and context as a result of research and cognitive activities in the process of examining their peculiarities"[6]..As the Swiss psychologist J. Piaget says in his famous work: "Knowledge is not a copy of reality. To know an object or an event is not 
simply to look at it and make a mental copy of it - to know an object is to act on it, to transform the object, and to understand the process of this transformation»[19, p.20].

\subsection{The research and educational activity of non-linguist students}

The current situation of FLT could be described in terms of a research educational model aimed at professional cross-cultural communication.

In accordance with the new provisions of the programme for FL education the student ought not only to formulate ideas in FL correctly, use regular speech patterns and terminology but also be aware of and ready to observe cultural traditions accepted in the country of L2.

It should also be noteworthy that scientific values which are independent of age, gender, personal, national or confessional traits are grounded on common principles and algorithms of scientific tradition in line with the objective picture of the world and of the man in the world agreeable with the current empirical data.

The research work of a future specialist in natural sciences involves developing functional explorer's skills as a universal means of discovery of the real world through raising motivation and personal autonomous activity in the process of education based on appropriation and structuring of obtained knowledge which is becoming new and personally valued.

The following functions of the academic research work could be highlighted in the tertiary professional education, i.e., raising the culture of professional research by cultivating analytical and forecasting abilities, developing intellectual and creative potential along with professional mastery. Students' research is implemented through the conventional scientific stages, namely, orientation, problem identification and reflection $[6,7,14]$.

In terms of methodology and pedagogy these stages might be presented in the formalized structured form as: 1) data diagnostics; 2) juxtaposition of initial data with the expected result; 3 ) setting the goal and providing its rationale for a certain period of time;4) positioning of a future specialist in a task-performing process; 5) the involvement of all students in carrying out tasks; 6) defining the content matter, methods and forms of implementation; 7) evaluation of the obtained data and its comparison with the planned results.

Apparently it could be noticed that these tasks coincide with the stages of a physical experiment, namely, setting the goal, formulating the hypothesis, the choice of an object, subject matter and methods; defining short-term and long-term objectives; fixation of changes occurred in the course of the experiment; analysis of the obtained results and their comparison with the hypothesis and goal; interpretation.

The constant demonstration of the common structure of professional work of an engineer, for example, and a scientist highlights the exploratory (creative) nature of a future job and at the same time its technological character. The other-didactic- aspect of the offered methodology is its interdisciplinary connection between the Humanities and profile subject which generally enhances the relationship between special and FL training of a future specialist taking into account both the specifics of a profile and the cognitive nature of an academic discourse in general.

\subsection{Cognitive nature of an academic discourse}

Numerous foreign and native studies have revealed some universal features of an academic discourse. It is generally accepted that discourse is one and undivided process of language performance and its product, that is, a written and an oral text, together with the dynamics 
of its generation and interpretation. The concept "an academic discourse" possesses some constant properties, i.e., substantive content, cohesion and coherence, subordination of secondary details to the main focus, accuracy and objectivity, the use of those linguistic means which correspond to the demands of academic communication $[6,13,14]$.

The above-said could be illustrated by the following examples. For instance, in terms of vocabulary it is the use of scientific terminology hardly known beyond a narrow circle of specialists (e.g. viewfinder, heptode, phantastron, driving-point impedance, plate-supply rectifier). As far as grammar is concerned science papers demonstrate a number of regular patterns: 1) simple two-member sentences with a compound predicate consisting of a linking verb and a nominal predicate; 2) attributive groups (e.g. open-loop output impedance); 3)operational verbs (e.g. perform, obtain, provide, give, involve, entail, imply, result in, lead to); 4) adverb substitutes for pre-positional expressions with nouns (e.g., "accurately" is substituted for "with accuracy"); 5) verbs are substituted by verbal adjectives with prepositions (to be conducive to, to be destructive of, to be incidental to, to be responsive to) [13].

As regards the cognitive status of discourse the research has revealed that its discursive function is closely tied with information processing and presentation of knowledge. The generation of discourse is a many-faceted integration of processes and systems of the world perception and linguistic representation of the results of the system work. This is a cognitive process which involves a unique way of acquiring knowledge of the world and its verbal representation. Before being passed in the act of communication information is conceptually processed and acquires a new form as new knowledge. Discourse is believed by scholars to be a cognitive phenomenon dealing with transfer of knowledge and, which is more, production of new pieces of knowledge.

For that reason students are offered to explore the object in question according to the following plan:

1. The goals of research.

2. The overview of publications.

3. The factual data on the research object (experiments, graphs, statistics).

4. Challenges, problems, possible solutions.

5. Hypothesizing.

6. Reflection.

7. Conclusions.

Such "algorithmization"("technicality") of research work by no means inhibits students' creative initiative. On the contrary, by completing consistently all listed stages students acquire the knowledge of their own. The teacher's assistance consists in regular consulting, repetition and consolidation of the learnt material, note and text correction.

Keeping this scheme in mind, the authors propose a teaching algorithm - the so-called teaching strategy whose goal is to analyze a true-to life professional situation, consider a variability of related issues, find a practical solution, choose the best solution, come to a conclusion through the discussion as a group.

\section{Method. The strategy of teaching FL to non-linguists}

In academic year 2019/20 the authors carried out a trial FLT course at the faculty of Physics and Maths of MGOU (Moscow Region State University) to test the theoretical considerations mentioned above. The strategy under investigation was approved by the University teaching staff while the students demonstrated positive dynamics in developing communicative competence, in particular, its discursive, sociolinguistic and strategic components. 
The course "The basics of professional communication in FL" is meant to take 72 academic hours and to comprise 3 stages which are described below.

\subsection{Three basic stages of teaching}

I. The first stage consists of searching and selecting universal speech patterns generally used in real-life academic communication, their classifying and forming ready-made matrices for different types of utterances: a report, a presentation, a seminar talk. To achieve this a number of word-transformation exercises are to be completed as well as stringing parts of a text together, selecting relevant sentences and, finally, compiling an autonomous text out of patterns tailored for speaker's needs. Generally the stage takes not less than 20-24 academic hours resulting in students' speech pro-duction of their own. The stage is aimed at encouraging students to build up confidence in managing tasks.

II. The second stage ("consolidation") is characterized by the considerate extension of speech patterns and situations. Students do the drilling of synonyms/antonyms of the learnt expressions, acquire new vocabulary and compile their own glossary of general and special terms. It is noteworthy that the compilation of a glossary is individual autonomous work since students acquire vocabulary through reading highly specialized literature. As far as grammar is concerned the use of different verb forms or modals is thoroughly studied at this stage, for example, in various contexts. Individual work starts to prevail and it takes the form of consultations about one's own texts. A teacher fosters the use of original sources and search for finding expressions and classifying them to be appropriated in further speech production. Micro-dialogues might also be acted out during the period. Taking 24 hours, this stage is marked by individualization.

III stage ("implementation") deals with putting acquired knowledge into practice. The risk of making mistakes is minimized by precedent encouragement and fostering of students at the first two stages. Students are to complete creative tasks such as making a presentation/report, writing a discursive essay or justifying/challenging one's position. As there was no feedback at the previous stages this one introduces question-answer exercises, making dialogues of one's own and preparing for taking part in discussions. The final testing might include participation in discussion about "Quantum computing", for instance. The role of the teacher involves grading lexical and grammar use, more or less subjective assessment of the content scientific relevance as well as students' negotiability and availability of general information.

\subsection{Discussion highlights in FL training}

The fragment of debates presented below on "Quantum computing" could serve as a demonstration of the FLT strategy.

The discussion on the issue is held in the form of debates. Students get acquainted with the topic in advance, the teacher explains the rules, time-limit and the highlights of the afore-coming game. In order to stir polemical spirit students are offered to read several problem-containing texts, watch films, consider several problems (a list is prepared by an English teacher) and an indefinite number of independent sources. It is up to students to choose one of the issues to speak about. The students' group is divided into 3 teams, that is, "optimists" (Group 1) who are to prove the significance of the discovery for mankind, "pessimists" (Group 2) who stand for the negative and harmful impact of the issue under discussion. Each of the teams is further subdivided into two groups. The first one prepares arguments, while the other deals with counter-arguments. The third group is "judges" (Group 3). The latter has to weigh the validity of argumentation and decide on the winner. As has been mentioned above, the preparative stage consists in analyzing related materials, 
looking for examples and building up argumentation (e.g. origin of the phenomenon of quantum computing, its founders, fields of science it could be used, its advantages and disadvantages, commercial availability of quantum computers, the equations of quantum mechanics, Heisenberg's uncertainty principle, physicists' current understanding of the issue, the notion of the Planck time, the repelling Lagrangian coherent structures, etc.).

The teacher of English, in his/her turn, provides the students with the necessary linguistic means and formulaic expressions (including specialized and academic vocabulary) which can help structure their utterances, following the scheme given in 2.3., namely:

- Einstein's general theory of relativity requires that...

- It was supposed that...

- The expectations for the fifth generation systems seem to require that...

- This led early string theorists to propose that...

- Theorists have determined that...

- The goal of Einstein's unified theory was to get a theoretical framework that...

- The book is mainly concerned with the problem of...

- We tried to find a new way of comparing the two phenomena.

- Our team came up with a solution to the problem of ...

During the debates each team presents their position, tries to prove the validity of their arguments, challenges the opponents' views, using the learnt word-combinations as in the following statements:

- Our group wanted to see if we could explain the phenomenon in another way...

- Our research questioned the notion that...

- The suggested methodology does not work in a number of cases...

- Unfortunately, our opponents did not have a detailed plan of investigation...

- We cannot fully agree with their standpoint for a number of reasons...

- We are totally opposed to the idea offered by Group 2.

Finally, the group of judges (Group 3) concludes the discussion giving their own viewpoints and proving their ideas with reliable data, facts, citing the words of outstanding scientists in the field, making perspectives of quantum computing and giving predictions about the future of the discussed phenomenon.

In the end the teacher assesses everyone's performance with a 100-point system and upon such criteria as topic relevance, creativity, consistency, adequacy, audience rapport, no dependence on notes, language (vocabulary, grammar, pronunciation), interaction with other participants, cheering and enthusiasm.

\section{Results and discussion}

In the context of research into the expediency of oral speech interaction in the form of a professional discussion a trial training was held for the students of Physics and Maths faculty of MGOU. The course involved developing the methodical recommendations for stimulating professional speaking activity in FL classes as well as two questionnaire surveys both initial and final.

The initial survey was aimed at disclosing students' views on pair (group, team) work at FL lessons. The target group comprised 34 second-year students who study English as a first or a second foreign language at MGOU. The survey revealed that the majority of students $(81 \%)$ preferred working in pairs/groups/teams in FL classes. The respondents answered that such work improved their dialogic and monologic as well as auditing skills $(83.6 \%, 51.8 \%$ and $44.3 \%$ respectively). However, the students were understood to have faced some difficulties in the course of intercommunication, namely, insufficient vocabulary $(47.5 \%)$, lack of relevant information $(35 \%)$, misunderstanding of partner's 
speech $(17.5 \%)$, mistakes in grammar usage (15\%), failure in expressing one's own opinion $(15 \%)$, incomplete knowledge of the subject matter $(5 \%)$.

The data of the survey provide evidence on the immaturity of strategic competence, the scarcity of background knowledge and the dearth of polemic skills. The former implies the compensatory techniques used in various situations of professional communication in case of language deficiency.

The results of the survey showed that the majority of the respondents $(96.5 \%)$ preferred working in homogeneous groups/teams with the students' equal level of language knowledge. This might be explained by the competitive spirit which arouses in these groups despite differences in linguistic competence. The students put it as follows, "It is better to be equal among equals than weaker or stronger than others."

In answer to the question about the advantages of speaking interaction in class the majority of students mentioned the proximity to the real-life communication $(75.6 \%)$, the diversity of educational tools $(71.5 \%)$, the additional peer communion $(37 \%)$, the elements of self-reflection.

The survey data taken into account, the electronic methodical memorandum was compiled with the scenario of "Quantum computing" discussion and a trial training was organized with a view to test students' verbal behaviour in simulated real-life situations, i.e., an international conference or a seminar.

The results of the trial training demonstrated that the students were interested in interactive forms of team work providing it was well-organized in FL class and adopted a creative approach. The freedom of choice in presentational forms of the learnt material (a presentation, a speech, a report, a brief statement or a replica) was proved to be of primary importance which had an immediate effect on the quality performance and the rise in inner motivation of both high- and low-achievers in language learning.

All of the aforesaid might be witnessed by the data of the second survey which was conducted in the same group at the final stage of working on "Quantum computing" issue. The majority of respondents $(87.6 \%)$ expressed their willingness to participate in further discussions in FL classes with many of whom (47.8\%) would prefer to have such lessons at least once a week. The evidence may lead to a conclusion that FLL motivation increases due to interaction in class.

In the reply to question about the effects of oral-speech interaction on learning the respondents answered that it brought a variety in the educational process (94\%), enabled them to use language in situations similar to real life (92\%), to play different parts $(64.2 \%)$, to communicate with peers in teams/groups $(66.1 \%)$, and, moreover, to practice verbal and nonverbal behavior in professional communication environment (33.2\%).

According to the survey, the problems faced by the students during the study included limited vocabulary $(80.1 \%)$, the unavailability of relevant information $(3.2 \%)$, inability to express one's viewpoint in a dialogue19.2\%), grammar difficulties (11\%), misunderstanding of the interlocutor's speech (15\%). Apparently, the extension of vocabulary remains the issue to be dealt with primarily for interaction in FL class.

In summing up the results, it may be concluded that in order to stimulate oral-speech interaction among students the wide range of communicative, edutainment, problemsolving as well as team training techniques are to be employed, some of which have been elaborated and demonstrated in the present study. Which is more, it is also worth mentioning, the closer to real life the situation recreated in class is the more stimulating it appears to students who become more willing and more prepared to acquire professional verbal and nonverbal skills and competences. The reconstruction of a true-to-life environment in FL class depends on teachers' and students' creative skills as well as on study and use of authentic materials and innovative information technologies. 


\section{Conclusion}

The research has revealed that many-sided communication implies the involvement of every participant including a teacher, parity, accounting for situations of professional information exchange and structuring of material. This form of communication at the lesson enables not only checking the prepared monological speech but also introducing the subject matter of a profile discipline.

The advantages of the FLT directed at open communication might be described as follows:

1. Students acquire knowledge better if they approach a discipline through their personal experience;

2. Students get more involved in educational activity;

3. Students perceive information with less effort if the former is structured and if a teacher takes into account their views which may not coincide with his/her personal opinion.

It is worth mentioning that many-sided communication is also an indispensable tool for constructing one's own knowledge.

Finally it may be stated that the strategy under consideration allows completing several tasks at one and the same time with developing professional communicative skills, being first among equals. It also enables participants to establish emotional rapport, to work in team, to account for a different point of view, and it also stimulates motivation, creativity and imagination, freedom of self-expression as well as mutual respect and democratic approach.

Just as importantly, the interactive form of classwork (in our case a discussion) described in the paper is directed at tighter interaction between students and a teacher, among students themselves, being student-oriented as a whole.

Summing up, it is worth mentioning that the proposed strategy goes back to the conventional linguodidactic and methodical approaches to teaching participation in professional debates, and mastering the professional speaking skills of future specialists.

\section{References}

1. P.G. Altbach, Studies in Higher Education 38(3), 316-330 (2013)

2. S. Guri-Rosenblit, H. Sebkova, U. Teichler, Higher Education Policy 20, 373-389 (2007)

3. S. Guri-Rosenblit, The European Higher Education Area. Springer, Cham (2015) https://doi.org/10.1007/978-3-319-20877-0_2

4. O. Klochkova, E. Komochkina, A. Mustafina, Procedia - Social and Behavioral Sciences 236, 271-276 (2016)

5. V. Naidoo, International Higher Education 58, 6-7 (2010)

6. L.V. Yarotskaya, Bulletin of Moscow State Linguistic University 16(755), 112-124 (2016)

7. A. Fisma, A. Pammu, R. Jubhari, ELS Journal on Interdisciplinary Studies in Humanities 4(1), 87-91 (2021) https://doi.org/10.34050/elsjish.v4i1.13366

8. V. Nursyarah, N.A. Drajati, N. Ngadiso, ELS Journal on Interdisciplinary Studies in Humanities 2(4), 582-594 (2019) https://doi.org/10.34050/els-jish.v2i4.8139

9. N. A. A. Jumrah, M. Azizah, ELS Journal on Interdisciplinary Studies in Humanities 4(1), 20-28 (2021). https://doi.org/10.34050/elsjish.v4i1.13360 
10. P. Berger, Th. Luckmann, The Social Construction of Reality. A Treatise in the Sociology of Knowledge (London, Penguin Books, 1991)

11. E.A. Komochkina, L.V. Yarotskaya, Higher Education in Russia 29(5), 64-72 (2020) DOI: https://doi.org/10.31992/0869-3617-2020-29-5-64-72

12. E. Komochkina, T. Selesneva, N. Akimova, The European Proceedings of Social and Behavioral Sciences EpSbs 98, 169-178 (2020)

13. A. Remache, International Journal of English Language Teaching 1(2), 37-52 (2013)

14. C. Cavage, University Success Oral Communication, Transition Level with MyEnglishLab (London, Pearson Education, 2017)

15. M. Heidegger, Sein und Zeit (Tubingen: Max Niemeyer Verlag, 2006)

16. D. Dorner, The Logic Of Failure: Recognizing And Avoiding Error In Complex Situations (New-York, Basic Books, 1997)

17. O.F. Klochkova, E.A. Komochkina, Insight into the Structure. Textbook for Physics Master Students (Moscow, NRNU MEPhI Publ., 2016)

18. M. McCarthy, F. O'Dell, Academic vocabulary in use (Cambridge, CUP, 2008)

19. J. Piaget, Development and learning. Piaget rediscovered. A report on the Conference on cognitive studies and curriculum development (Ithaca (NY), Cornell University Press, 1964) 